\title{
The Effect of Travel Blog Quality on User Satisfaction and Intention to Revisit
}

\author{
Hwa-Yeol Choi ${ }^{1}$ and Yoo Kyung Lee ${ }^{2 *}$ \\ ${ }^{1}$ Professor, Dept. of Aviation Service Management, South Korea \\ ${ }^{2}$ Hanyang University Graduate School of Tourism, Master's Program, South Korea

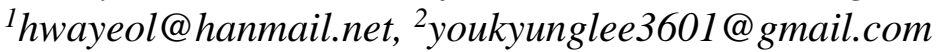

\begin{abstract}
Blogs have emerged as a new form of sharing information over the Internet. In other words, blogs are made more comfortable and useful communication tools in that they have the characteristics of public disclosure of personal opinions and information, which allows users to have two-way communication channels compared to other media, and are used to communicate with others compiling scattered information. Through this, the researchers focused on travel blogs that have been highly praised for the emergence of the Work-Life Balance generation. Despite the considerable increase in the function and influence of travel blogs, there has been little previous research into the quality envisioned by blogs and users. Therefore, the study studied how the quality of the travel blog affects visitors' satisfaction and willingness to revisit through a causal research model. Based on previous research on the quality of tourist information websites, the factors of quality were verified and the availability was discussed through this paper. In addition, the study conducted an online survey of those who said they had used blogs. As a result, the positive relationship between the quality of the travel blog and the user satisfaction, user satisfaction and willingness to revisit as well as the quality and intention of the travel blog was confirmed. This shows that we have to make efforts to further improve the quality of our travel blog. It leaves implications for related research such as how to improve travel blog quality later on.
\end{abstract}

Keywords: Blog, Quality, Satisfaction level, Revisit intention, Travel

\section{Introduction}

It is an information age in which information can be quickly moved across borders, created wealth and shared free of charge through the Internet. Blogs are individual media, different from homepages and communities. Blog usability is more pronounced in that it is used to integrate distributed information and communicate with people around it [1]. While the types of blogs vary depending on the topic, the study is aimed at studying blogs related to travel, which are becoming increasingly important as quality of life and leisure activities draw attention. As Korea's lifestyle changed, people's interest in travel increased, and they wanted a lot of information about travel to improve their quality of life, blogs were created to search for information about travel experiences through other people's blogs through Internet information exchange. Photos, travel information and reviews provided by blogs are important tools for selecting travel destinations, and unlike travel agencies and other

Article history:

Received (June 3, 2020), Review Result (July 4, 2020), Accepted (August 9, 2020) 
professional companies' websites, users can trust information from public bloggers by making them feel homogeneity and intimacy. It serves as a blogger who has been to inform others of his experiences, such as the pros and cons of the destination, through his blog, and potential travelers seem to be in the process of contacting various blogs to find the information they want. People are easily sharing, comparing and acquiring various information related to travel through various personal blogs. Photos, travel destination information and reviews provided by blogs are becoming important tools for choosing destinations, and unlike websites of professional companies such as travel agencies, information from bloggers is gaining trust by making users feel homogeneity and intimacy. This serves as a bloggers who have been to any travel destination inform other people of their experiences, such as the advantages and disadvantages of the destination, through their blogs, and potential travelers are exposed to many blogs to find the information they want. Despite the increased role of travel blogs, however, research is rare on the quality of blogs, namely how to effectively operate blogs and evaluate the effectiveness of existing blogs. A prior study on the quality of blogs is [2] looking at the impact of blog service quality on service value and customer satisfaction, but this suggests the strategy of portal sites that operate blog services. Since there was no prior study of blog quality from the perspective of bloggers and users, this study found the quality factors of travel blogs based on the quality dimensions of tourism information websites [3][4], and [5], and examined the effect of travel blog quality on users' satisfaction and intention to revisit through a causal research model.

\section{Theoretical background}

\subsection{Blog concepts and characteristics}

Blog is short for weblog, which combines web, which means the Internet, and log, which means data, and is created as a personal diary that is frequently updated to freely express one's thoughts, arguments, and daily life through the web. In addition, communication functions have been strengthened to serve as a tool for building a strong social-network on the Internet, enabling one's experience and thoughts to accumulate and be shared as information and grow into a vast information network. Blogs are one-man media in that they can express their opinions in various forms, such as diaries and column articles, while delivering their opinions to an unspecified number of people through the network characteristics of blogs. Johnson and AMP; Kaye defined blogs as 'a diary-style website where individual observations and news are recorded in the order of posting [6], along with comments and links related to them.' In other words, blogs can be defined as media that allows an individual to become the main body and share his or her own created and processed content with the public in the form of a diary. In addition, Gumbrecht is a study that deals with blogs as a new medium, evaluating blogs as a communication medium that can replace social interaction due to their two-way nature [7], while Herring et al. says blogs are both excellent interactive tools [8] and a communication tool suitable for self-expression [9]. These studies talk about the possibility that blogs will replace existing communication channels. Blogs are frequently updated with recent data organized in chronological order, and titles and texts are also exposed on the first screen. Also, each post is reflected at the same time as it is written, and each post has a unique address, and is woven like a net through a trackback. Trackback is one of the main functions used in blogs, which is simply to automatically generate reverse links, which is the ability to write remote comments and inform them. This serves to create a communication network by creating a link between blogs that are strictly one-person media 
oriented, and after reading someone's blog and writing their opinions about it on their blog, exchanging trackbacks, links to new postings are attached under the original text. Blogs have the characteristics of their content, are less editable and more public than traditional Internet postings, and are capable of communicating one-on-one, one-to-many, and many-to-many. In addition, the biggest technical characteristic of blogs is the convenience of operating their own blogs by entering websites such as portals, signing up for membership, and entering a few options. Combining these characteristics of blogs, blogs can be defined as media [10] in which an individual can freely create, collect, and process content he or she wants in the form of a diary and share it with acquaintances around him or the anonymous public. Blogs can be distinguished from existing Web communities in terms of 'personality' in which one individual becomes the main agent of operation, and can be differentiated from existing websites in terms of 'connectivity' that they are linked in various ways with other bloggers. The variety of information delivery features and unique characteristics of blogs suggest that blogs can be used as a more efficient tool for information delivery beyond the constraints of web pages that deliver information in a relatively one-way direction [11] and the format and content aspects of blogs make the existing web an important marketing tool in terms of the strong tendency to remeasure [12]. The tourism industry also recognizes the importance of Internet marketing using blogs, and in particular, the images provided by blogs can be used as a communication tool to connect potential travelers and destinations by recording memories of experience and providing users with prior information through photos, and turning potential travelers into actual travelers [13] Travel blogs have the advantage of securing the credibility of blog users in that information providers are ordinary people, not providers of services or product sellers. Given that the information provider is an ordinary person and does not have a specific purpose, it has the advantage that users can feel homogeneity and intimacy, and that they can easily sympathize with the information provider's experience by visiting with the purpose of sharing hobbies or learning other people's opinions rather than doubts about information.

\subsection{Travel blog quality}

The information provided by travel blogs has become an important tool in selecting destinations, and it is necessary to measure the quality of travel blogs in a situation where users want to gain trust. Websites that contain useful information and additional benefits available to consumers can help improve tourism reputation and increase sales. However, users of the tourism information website are composed of various stakeholders, and since they have opposite goals, it is very difficult to measure the quality of the website [14]. There is a need for a model that directly targets interaction and technology that can reflect the characteristics of a website. In addition, there are existing researches to measure website quality and customer [15], but it is necessary to identify factors that determine the overall quality of websites and empirical analysis of the relationship between them [16]. Existing studies have applied research results to the tourism sector, but most studies have limitations that are limited to specific industries such as hotels and lodging agencies, and travel agencies. There is a growing need for research based on research [5]. In particular, the tourism information website needs to be viewed from the viewpoint of integrating each individual sector of the private or public sectors, reflecting the characteristics of handling heterogeneous complex products and service products. Therefore, in this study, based on the results of [5], the quality of tourism website was divided into four dimensions: information quality, service 
quality, system quality, and interest quality. We examined the effect of the quality of a travel blog on the satisfaction of users and the intention to revisit.

\subsection{Satisfaction of travel blog users and intention to revisit}

Satisfaction is subjective because it is perceived differently by each person to the extent that the individual feels utility. Blogging allows users to express satisfaction with the difference in the expected effects and experience of the time spent on blog visits. In other words, the subjective psychological state resulting from comparing the level of user expectations with the level of awareness of the actual experience in a travel blog visit is satisfactory. In the end, satisfaction can be said to be determined by the results of subjective judgments about previous expectations and experiences, which can be assessed on a judgment basis through personal feelings [17] which can be determined by psychological factors [18]. The definition of user satisfaction is classified according to whether it is based on one-time experience or multiple iterative experiences. In terms of one-time transactions, user satisfaction looks at transactions from a specific perspective. From the perspective of multiple iterative transactions, it is defined as the cumulative user satisfaction called assessment based on experience accumulated over time [19]. Based on this definition of user satisfaction, the satisfaction level of travel blog users can be defined as 'the result obtained from the experience of blogging or from the satisfaction of the purpose of use'. The satisfaction level of travel blog users is affected by various factors, such as blog images and users' feelings, but especially by the qualitative perception of services encountered while visiting the travel blog. Therefore, research on factors affecting the satisfaction of travel blogs should measure the quality components of blogs and determine the impact of these quality factors on satisfaction. Many studies have been conducted on the satisfaction of website users and their intention to revisit. Studies have shown that the higher the satisfaction level, the more positive the intention of revisiting. The higher the satisfaction level of using the website, the higher the willingness to revisit [20][21][22]. In particular, Chen and AMP; Wells looked at the impact of travel blog users' satisfaction on their willingness to revisit through research results on website satisfaction and intention to revisit [23]. Attitudes toward websites are important decisions for users to use websites, such as willingness to revisit websites show one's human nature In other words, the higher the user's satisfaction with the website, the more willing they are to revisit the website.

\section{Research model design and method}

The questionnaire used in this study is based on prior research on travel blogs mentioned in the theoretical background and prior study on their satisfaction and intention to revisit, consisting of 21 questions about travel blog quality characteristics of users of travel blogs, 2 questions related to usability, and 7 questions on demographic characteristics to identify respondents' characteristics. The form of the questionnaire was measured using nominal measures for demographic details, and the questions related to travel blog quality characteristics, usability, and revisit intentions were measured using the Likert 5-point scale

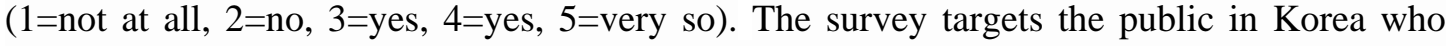
visit travel blogs, and the sampling is based on the "Convenience Sampling" of nonprobability sampling. In particular, considering difficulties in collecting data and the expected number of appropriate cases, blog-related lectures were taken at the lifelong education centers of two universities in Seoul, and surveys were conducted for two weeks from December 16, 2019 for ordinary people with experience using travel blogs. A total of 250 copies were 
collected for the method of filling out the questionnaire using the self-administered QSM, but a total of 197 copies were analyzed using data, except for 13 copies that could not be used as data due to insincere responses or missing parts of the survey. To carry out this study, frequency analysis was performed to identify demographic characteristics using the SPS statistical package, reliability and validity analysis, and reliability analysis, factor analysis, and multiple regression analysis were performed for the test of the research theory.

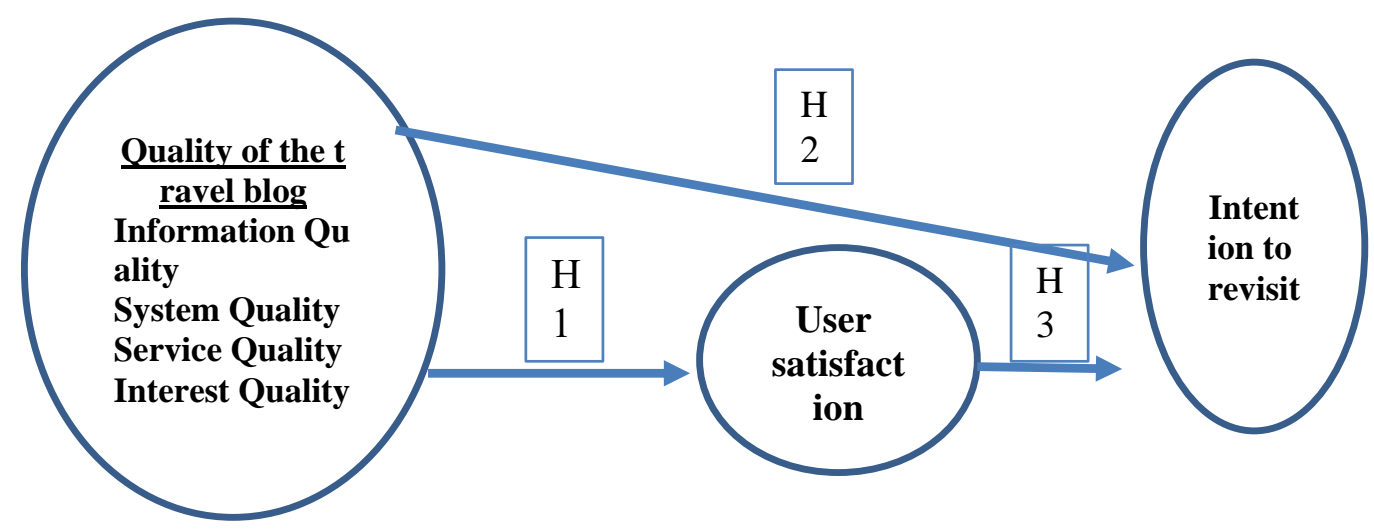

Figure 1. To clarify the relationship between the quality of the travel blog, user satisfaction, and intention to revisit, and summarized the research hypothesis according to $\langle\mathrm{H} 1\rangle$ to $\langle\mathrm{H} 3\rangle$

$<\mathrm{H} 1>$ Travel blog quality will have a positive impact on user satisfaction.

$<\mathrm{H} 2>$ The quality of the travel blog will have a positive effect on the intention to revisit. $<\mathrm{H} 3>$ User satisfaction will have a positive effect on the intention to revisit.

\section{Results}

\subsection{Travel blog quality will have positive impact on user satisfaction}

In the regression model, the $\mathrm{F}$ value was 61.456 , which was statistically significant at the significance level of .001 , and $56.2 \%$ of the total variance was explanatory.

Table 1. Result of Hypothesis 1

\begin{tabular}{|c|c|c|c|c|c|}
\hline \multirow{2}{*}{ Factors } & \multicolumn{2}{|c|}{ Non-standardization factor } & \multirow{2}{*}{$\begin{array}{c}\begin{array}{c}\text { Standardization } \\
\text { Factor }\end{array} \\
\text { Beta }\end{array}$} & \multirow{2}{*}{$\mathrm{t}$} & \multirow{2}{*}{$\mathrm{P}$} \\
\hline & B & Standard Error & & & \\
\hline Invariable number & -.155 & .277 & & -.559 & .577 \\
\hline Information diversity & .204 & .071 & .194 & 2.871 & $.005^{* *}$ \\
\hline Interest & .266 & .075 & .216 & 3.531 & $.001 * *$ \\
\hline Service & .345 & .084 & .265 & 4.107 & $.000 * * *$ \\
\hline System & .318 & .070 & .287 & 4.540 & $.000 * * *$ \\
\hline Information accuracy & .005 & .075 & .005 & .072 & .942 \\
\hline Usability & -.034 & .061 & -.030 & -.562 & .575 \\
\hline \multicolumn{6}{|c|}{$\begin{array}{l}R 2=.562 \text { Adjusted } R 2=.548 F=61.456 p=.000 \text { Durbin-Watson }=2.202 \\
* P<0.05, * * P<0.01, * * * p<0.001\end{array}$} \\
\hline
\end{tabular}




\subsection{The quality of the travel blog will have a positive effect on the intention to revisit}

In the result of t-testing for the regression coefficients of the attributes input to the regression equation, the interest quality, system quality, and service quality were statistically significant at the significance level of .05.

Table 2. Result of Hypothesis 2

\begin{tabular}{|c|c|c|c|c|c|}
\hline \multirow{2}{*}{ Factors } & \multicolumn{2}{|c|}{ Non-standardization Factor } & $\begin{array}{c}\text { Standardization } \\
\text { Factor }\end{array}$ & \multirow{2}{*}{$\mathrm{t}$} & $\mathrm{P}$ \\
\cline { 2 - 5 } & $\mathrm{B}$ & Standard Error & Beta & & \\
\hline Invariable number & .175 & .327 & & .536 & .592 \\
\hline Information diversity & -.036 & .084 & -.034 & -.432 & .666 \\
\hline Interest & .468 & .089 & .377 & 5.256 & $.000 * * *$ \\
\hline Service & .188 & .099 & .144 & 1.899 & $.049 *$ \\
\hline System & .260 & .083 & .233 & 3.140 & $.002 * *$ \\
\hline Information accuracy & .030 & .089 & .027 & .339 & .735 \\
\hline Usability & .068 & .072 & .060 & .944 & .346 \\
\hline $\begin{array}{l}R 2=.398 \text { AdjustedR2 }=.379 F=20.955 p=.000 \text { Durbin-Watson }=2.138 \\
* P<0.05, * * P<0.01, * * * p<0.001\end{array}$ & & & & \\
\hline
\end{tabular}

\subsection{User satisfaction will have a positive effect on the intention to revisit}

The regression model showed a value of 283.806 , which was statistically significant at the significance level of .01, and it was found to have an explanatory power of $59.3 \%$ of the total variance. The higher the user satisfaction, the higher the revisit intention.

Table 3. Result of Hypothesis 3

\begin{tabular}{|c|c|c|c|c|c|c|}
\hline \multirow{2}{*}{$\begin{array}{l}\text { Dependent } \\
\text { variables }\end{array}$} & \multirow{2}{*}{ Independent variables } & \multicolumn{2}{|c|}{ Non-standardization factor } & \multirow{2}{*}{$\begin{array}{l}\text { Standardization } \\
\text { Factor } \\
\text { Beta } \\
\end{array}$} & \multirow[t]{2}{*}{$\mathrm{t}$} & \multirow[t]{2}{*}{$\mathrm{p}$} \\
\hline & & B & Standard Error & & & \\
\hline \multirow{2}{*}{$\begin{array}{l}\text { Intention to } \\
\text { revisit }\end{array}$} & Invariable (number) & .748 & .166 & & 4.508 & .000 \\
\hline & User satisfaction & .775 & .046 & .770 & 16.847 & $.000 * * *$ \\
\hline
\end{tabular}

\section{Conclusion}

This study looked at travel-related blogs, whose importance has been increasing as quality of life and leisure activities have been noticed, and examined the relevance and influence between the quality of travel blogs, user satisfaction, and the intention of revisiting. Despite the increased role of travel blogs, in the absence of prior research on the quality of blogs from the perspective of bloggers and users, this study found the quality factors of travel blogs based on the quality dimensions of preceding studies on the quality and satisfaction of tourist information websites, revealed their usefulness, and examined the impact of travel blogs' quality on users' satisfaction and intention to revisit through a causal research model. The analysis revealed that there is a positive relationship between the quality and user satisfaction of the travel blog, the satisfaction of the users and the revisit, and the quality of the travel blog and the revisit. Specific results and implications of this study are as follows. 
First, six components of travel blog quality were derived, unlike the information, system, service, and quality of interest on tourist information websites, such as information diversity quality that indicates diversity and usefulness of information and content, quality of service that causes fun and enjoyment, quality of system that indicates speed of access and design, accuracy of information and content, quality of information that provides the latest information, and easy-to-use usability quality. These results can be interpreted as consisting of more diverse factors than tourist websites, depending on the characteristics of blogs operated on information provided by service providers such as travel agencies or non-product sellers. Second, the quality of travel blogs affecting user satisfaction was shown by systems, services, interests, and information diversity quality, among which the system quality representing access speed, page loading speed, design, etc. was the most influential. The travel blog provides continuous service through a concise design, indicates that the factors causing interest and the need for a variety of information and content. Third, the effect of travel blog quality on revisit intention was found that interest, system and service quality had a positive effect, and the interesting quality factor that caused pleasure and fun was the biggest influence. These results suggest that bloggers who run travel blogs should pay attention to the speed and design aspects of the site, quick feedback on questions, and factors that cause fun and pleasure to differentiate themselves from other blogs, as well as various and accurate information. However, this study conducted a survey for those who have experience using travel blogs in the process of collecting research subjects, and in future studies, it would be more practical to conduct online surveys for those who used blogs directly in conjunction with bloggers who run travel blogs. Further research may also be meaningful to divide the different types of travel blogs and look at their respective quality.

\section{References}

[1] Moon Jeong-hoon, "The effect of the system quality perceived by blog users on customer loyalty, e-business research," vol.6, no.3, pp.95-118, (2005)

[2] Cho Chul-ho and Kang Byeong-seo, "The effect of blog service quality on service value and customer satisfaction: focused on cyworld," Journal of Quality Management, vol.35, no.1, pp.35-51, (2007)

[3] Kim Jung-hoon, "The effect of city tour service quality on tourist satisfaction and behavioral intention, tourism research," vol.44, no.3, pp.1-20, (2019)

[4] Jung Nam-ho, Um Tae-yee, and Koo Chul-mo, "Factors affecting the value and satisfaction of online travel agency visitors: Focusing on the moderating effects of long-term orientation," Korean Journal of Hospitality and Tourism, vol.27, no.2, pp.1-19, (2018)

[5] Doo-Kyung Kim, Sungsoo Jang, and Jaeeun Kang, "A study on the quality evaluation of tourist information websites: focusing on visitors to jeju area," Tourism and Leisure Research, vol.18, no.3, pp.311-325, (2006)

[6] Johnson T. J. and Kaye B. K., "Wag the blog: How to reliance on traditional media the internet influence," Journal and Mass Communication Quarterly, vol.81, no.3, pp.622-642, (2004)

[7] Gumbrecht M., "Blogs as a protected space. WWW 2004 Workshop on the Weblogging Ecosystem: Aggregation, Analysis and Dynamics, (2004)

[8] Herring S., Scheidt L., Bonus S., and Wright E., "Bridging the gap: A genre analysis of weblogs, Proceedings 37th Annual HICSS Conference," Big Island, Hawaii, (2004)

[9] Han Sun, "A study on the aspects and implications of meaning practice through blog," Korean Journal of Journalism, vol.50, no.5, pp.354-383, (2006)

[10] Kim Kyung-hee and Bae Jin-ah, "Blogging communications research by bloggers in their 30s," Journal of the Press of Korea, vol.50, no.5, pp.5-29, (2006) 
The Effect of Travel Blog Quality on User Satisfaction and Intention to Revisit

[11] Kwon Sang-hee, "Blog style: research on genre type analysis according to communicator, composition, content, and connectivity," Cyber Communication Journal, vol.15, pp.93-134, (2005)

[12] Kwon Sang-hee and Woo Ji-soo, "Blog media research: a study on blog usage and satisfaction and cognitive behavior," Korean Journal of Broadcasting, vol.19, no.2, pp.419-460, (2005)

[13] Lin, Y. S. and Huang, J. Y., "Internet blogs as a tourism marketing medium: A case study, Journal of Business Research,” vol.59, pp.1201-1205, (2006)

[14] Lee Mi-hye and Lee Soo-jin, "Research on tourism information website evaluation, cultural tourism research," vol.4, no.2, pp.199-221, (2002)

[15] Palmer J. W., "Web site usability, design, and performance metrics, Information Systems Research," vol.13, no.2, pp.151-167, (2002)

[16] Liu L. and Arnett P., E”xploring the factors associated with Web site success in the context of electronic commerce," Information and Management, vol.38, no.1, pp.23-33, (2000)

[17] Kyunghee Lee, "Analysis of service quality evaluation of festivals: Focusing on the Hanbat Cultural Festival in Daejeon," Cultural Tourism Research, vol.5, no.2, pp.29-33, (2003)

[18] Jang Hye-won and Oh Sang-hoon, "A study on the self-monitoring and ethical tourism behavior according to the consumption values of tourists," Tourism and AMP, Leisure Research, vol.32, no.1, pp.53-74, (2020)

[19] Cho Kwang-haeng and Bong-kyu Park, "A study on the influence of conversion barriers and customer satisfaction on store loyalty, business studies," vol.28, no.1, pp.127-149, (1999)

[20] Ji-Hwan Yoon, So-Yeon Choi, Je-Jeon Park, and Chun-Kon Kim, "The effect of internet service quality of travel agencies on customer satisfaction and customer loyalty,” Tourism Research Journal, vol.20, no.2, pp.201-216, (2006)

[21] Sam-Kwon Jung, Hyun-Sook Seo, and Jung-Woo Byun, "The effect of organizational public relations of travel agency website users on customer satisfaction and loyalty," Tourism Research Journal, vol.21, no.2, pp.19-37, (2007)

[22] Lee Joo-hyun and Choi Young-gyun, "The impact of interaction on search engine websites on the consumer's involvement and attitude toward the site, intention to revisit the site, and research in advertising," vol.13, no.5, pp.223-247, (2002)

[23] Chen Q., AMP, and Wells W. D., “Attitude toward the site,” Journal of Advertising Research, vol.39, no.5, pp.27-37, (1999) 